\title{
Linear Polarized Matched-Input Active Integrated Transmit Antenna
}

\author{
Kimberley W. Eccleston \\ Dept of Electrical and Computer Engineering \\ University of Canterbury \\ Christchurch, New Zealand \\ E-mail: kim.eccleston@canterbury.ac.nz
}

\begin{abstract}
This paper shows the development of an active integrated transmit antenna which has linear polarization and a matched input. The active antenna comprises a rectangular microstrip patch that is fed by two FET s directly connected to its non-radiating edge. The FET s are driven equally but with a 180 degree phase difference by a modified branch-line coupler. Simulations of a $2.45 \mathrm{GHz}$ active integrated antenna demonstrate the feasibility.
\end{abstract}

Keywords- active integrated antennas; microstripline antennas; patch antennas; microwave amplifiers; branch-line couplers.

\section{INTRODUCTION}

Active integrated antennas integrate active components with the antenna so that the antenna serves other functions in addition to radiation. By integrating a power amplifier with an antenna, transmission lines and output matching circuits between antenna and the FET can be eliminated thereby reducing size and loss [1]. In this work we consider the additional role of 2 -way power combining. Active integrated transmit antennas that combine two FETs can either use a printed Yagi antenna [2] or a 2-port rectangular microstripline patch [3] - [5]. Since microstripline is used extensively in microwave circuits, it is advantageous to use microstrip patch antennas

Typically when a 2-port rectangular patch is used in a twoFET active transmit antenna, each FET drives a radiating edge of the patch, and the FETs are driven equally but 180 degrees out of phase by a rat-race coupler [3][4]. The dimensions of the patch are chosen to excite the TM010 resonance mode [6], giving rise to a linear polarization. Since they are fed at the radiating edges, some of the active circuit has to be located away from the patch, necessitating significant lengths of microstripline between the active circuit and antenna, so that antenna performance is not compromised. It has been shown that linear polarization can be achieved with multiple FETs driving a non-radiating edge of the patch [7], and this means that the circuitry can be placed immediately beside the antenna with minimal effect on the antenna characteristics, thereby eliminating extra microstripline.

A rat-race coupler will only have a matched input port if the other three ports are match terminated; requiring input matching for the FETs of an active transmit antenna. At low frequencies, the VSWR of the input of a FET is very high, and matching, if at all practical, is highly sensitive to FET parameter variations. Quadrature power dividers, such as the branch-line coupler, can achieve a matched input port without matched terminations at the output ports. The only requirement is that the terminations are identical and that the isolated port is match terminated. However, the two output signals will be in quadrature phase which is more suited for exciting a circularly polarized wave [6]. In a circular patch with correct feeding, both left-handed and right-handed circularly polarized waves can be excited resulting in net linear polarization [8]. However, this method requires the transistors to be precisely identical.

In this work, a modified branch-line coupler is used to obtain equal anti-phase input signals for the FETs which drive the non-radiating edge of a rectangular patch and establish linear polarization. The input port is matched.

\section{OVERVIEW OF PROPOSED ACTIVE ANTENNA}

Fig. 1 shows a general schematic of the proposed active antenna and includes voltage waveforms at the gate and drain of each FET. The input feeding network - the modified branch-line coupler - excites both FETs equally but with 180 degree phase difference. The idea is to excite the TM010 resonance mode of the antenna [6].

The antenna ports spaced $d$, are located symmetrically on the non-radiating edge, and $L$ is approximately half guidewavelength at the centre frequency, so that equal but anti-phase excitation of the ports will establish the TM010 resonance mode. Under this condition: radiation occurs at the edges of width $W$; linear polarization is achieved; and an electrical wall runs down the mid-line of the antenna and this allows the FET drain bias to be applied as shown. The port spacing $d$, and radiation edge width $W$ primarily determines the patch port impedance, whilst $L$ primarily determines the antenna centre frequency.

Fig. 2 shows the evolution of the modified coupler from the conventional branch-line coupler. For a conventional branchline coupler (Fig. 2(a)), a wave incident on port 1 will emerge at ports 2 and 3 with equal magnitude but with the wave em erging at port 3 lagging that at port 2 by 90 degrees. Waves reflected from port 2 and 3 terminations will appear at both ports 1 and 4 . But provided that these terminations are identical, and port 4 is match terminated, the reflected waves at port 1 will cancel and port 1 will appear matched. 


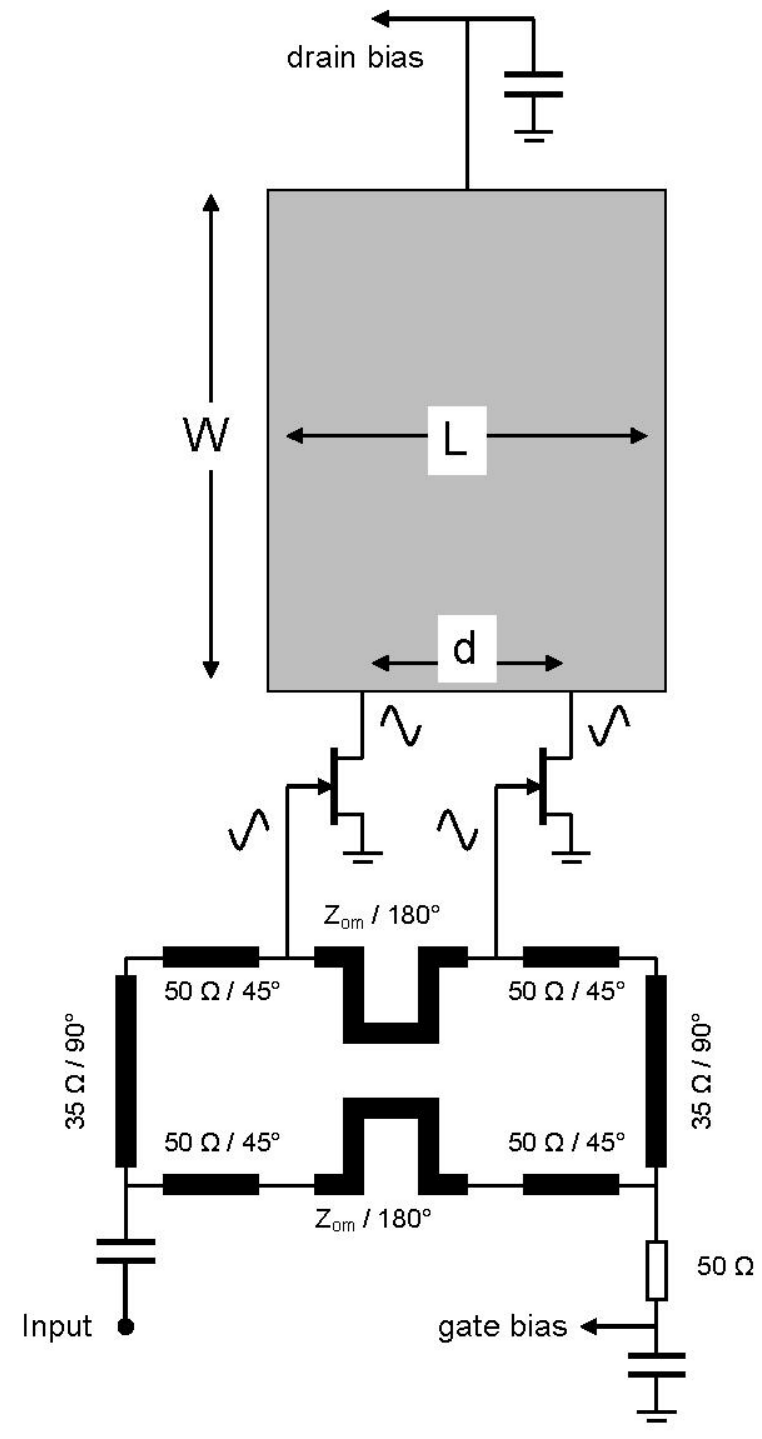

Fig 1. Schematic for proposed 2-way combining matched active antenna showing voltage waveforms at gate and drain of each FET. The capacitors are large valued for $\mathrm{DC}$ decoupling. The rectangular microstripline patch antenna is shown shaded grey.

If 180 degree transmission lines are inserted in the middle of the $50 \Omega$ lines as shown in Fig. 2(b), even and odd mode analysis [9] shows that the modified branch-line coupler (Fig. 2(b)) works exactly the same as the conventional branch-line coupler (Fig. 2(a)) at the centre frequency for an arbitrary value of $Z_{o m}$. The only difference being that the wave emerging at port 2 lags that at port 3 by 90 degrees. The modified branchline coupler therefore has a matched port 1 for identical mismatched port 2 and port 3 terminations. A distinction of the modified coupler is that the voltages at nodes $A$ and $B$ have identical magnitudes but have 180 degree phase difference.

The modified coupler is used in the active antenna as follows: ports 2 and 3 are open-circuit terminated, nodes $A$ and $B$ are connected to the gates of the two FETs, and the input capacitances of the FETs are parallel resonated with inductive shunt stubs. Parallel resonance of the FET inputs is less sensitive to parametric variation compared to matching and yet is sufficient to ensure proper operation of the coupler. Using this approach, the active antenna input port is matched and the FETs are driven equally but with 180 degree phase difference. The standing-wave on loop transmission structures can be decomposed into anti-clockwise and clockwise waves and means that the voltages at nodes $A$ and $B$ are sums of forward and a backward traveling waves, and this is beneficial in the presence of line losses [10].

(a)

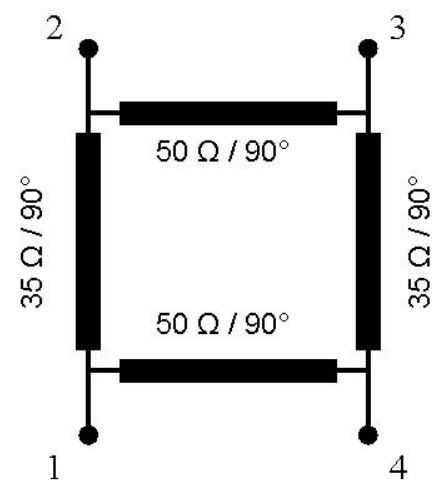

(b)

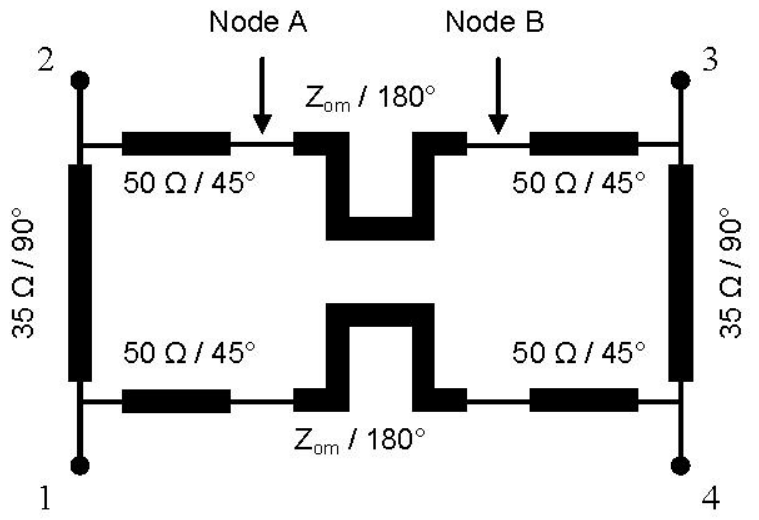

Fig 2. Evolution of the modified branch-line coupler (b) from the conventional branch-line coupler (a).

\section{ANTENNA DESIGN AND SIMULATION}

A substrate with dielectric constant of 2.22 and a thickness of $0.7874 \mathrm{~mm}$ was used. The antenna was designed to operate in the $2.45 \mathrm{GHz}$ band and this is achieved with $L$ equal to 40 $\mathrm{mm}$. $W$ was chosen to be $60 \mathrm{~mm}$ which ensures a useful value of radiation conductance, as well as avoided spurious modes around $2.45 \mathrm{GHz}$. Since the FET drains are to be connected directly to the antenna, it makes sense to use Z-parameters [7]. With $d$ set to quarter guide-wavelength, it can be shown, using the transmission line model of the antenna [6], $Z_{11}$ and $Z_{22}$ of the antenna are approximately half that when the ports are located on the radiating edges.

Method-of-Moment (MoM) EM simulations of the antenna were performed using commercially available software. The simulation domain was bounded by perfect electric conductors for the sides and bottom, and $377 \Omega /$ square resistive sheet for the top. To minimize coupling between the antenna and 
simulation boundary, the simulation domain size was $600 \mathrm{~mm}$ by $250 \mathrm{~mm}$ with a height of $60 \mathrm{~mm}$. The simulation grid size was $1 \mathrm{~mm}$ by $1 \mathrm{~mm}$.

Fig. 3 shows $Z_{11}$ as a function of frequency, and noting $Z_{22}$ and $Z_{11}$ are equal due to symmetry. It was found that the frequency response was independent of the termination connected to the drain bias connection of the antenna. The range of the real part of $Z_{11}$ is useful for medium power FETs. The antenna centre frequency is $2.46 \mathrm{GHz}$.

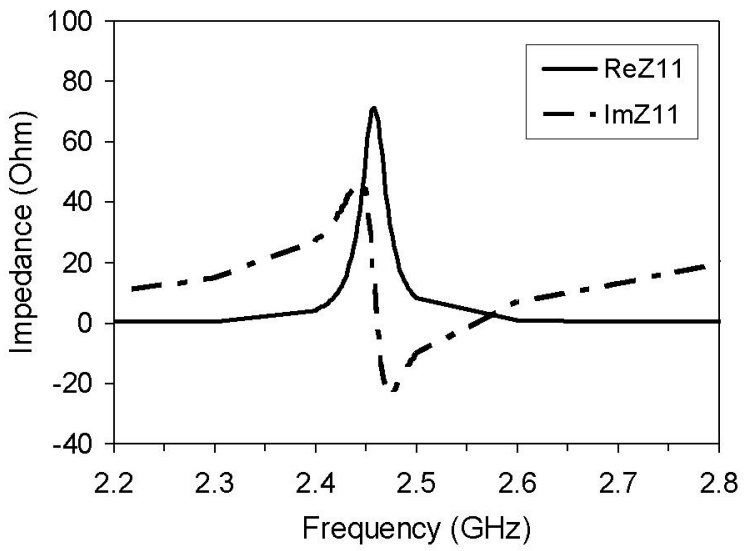

Fig. 3. Calculated frequency response of $Z_{11}$.

Fig. 4 shows the surface current density distribution on the antenna when the ports are equally driven but with 180 degree phase difference. This shows that the TM010 mode has been excited. Fig. 5 shows the radiation pattern for the same excitation condition as Fig. 4 and shows that linear polarization has been obtained. Other simulations were done with a via-toground in place of the drain bias connection to confirm isolation of the drain bias connection with RF operation.

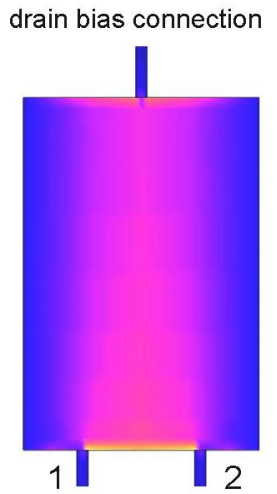

Fig. 4. Calculated surface current density at $2.45 \mathrm{GHz}$ (in color).

\section{Active ANTENNA SimUlation}

Fig. 6 shows the layout of the completed active antenna including the modified branch-line coupler and uses a pair of Eudyna FLK017WF GaAs Power FETs, and SMT0603 surface mount $10 \mathrm{pF}$ capacitors and $50 \Omega$ resistor. As $Z_{\text {om }}$ can be arbitrary, the width of the 180 degree lines of the modified branch-line coupler were set to $1 \mathrm{~mm}$ to allow them to be meandered to fill the coupler interior. Short-circuit stubs were connected at the gates of the FETs to parallel resonate the FET inputs. The layout shows that the active antenna is compact. Other work [7] has shown that circuitry close to the nonradiating edge of a rectangular patch has minimal effect on antenna performance - with at most a slight deterioration of the cross-polarization isolation.

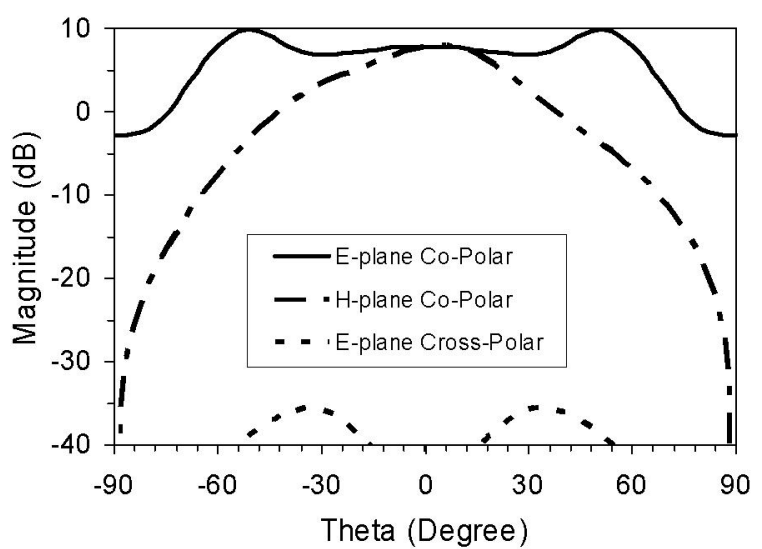

Fig 5. Calculated radiation pattern at $2.45 \mathrm{GHz}$. H-plane cross-polarization was negligible.

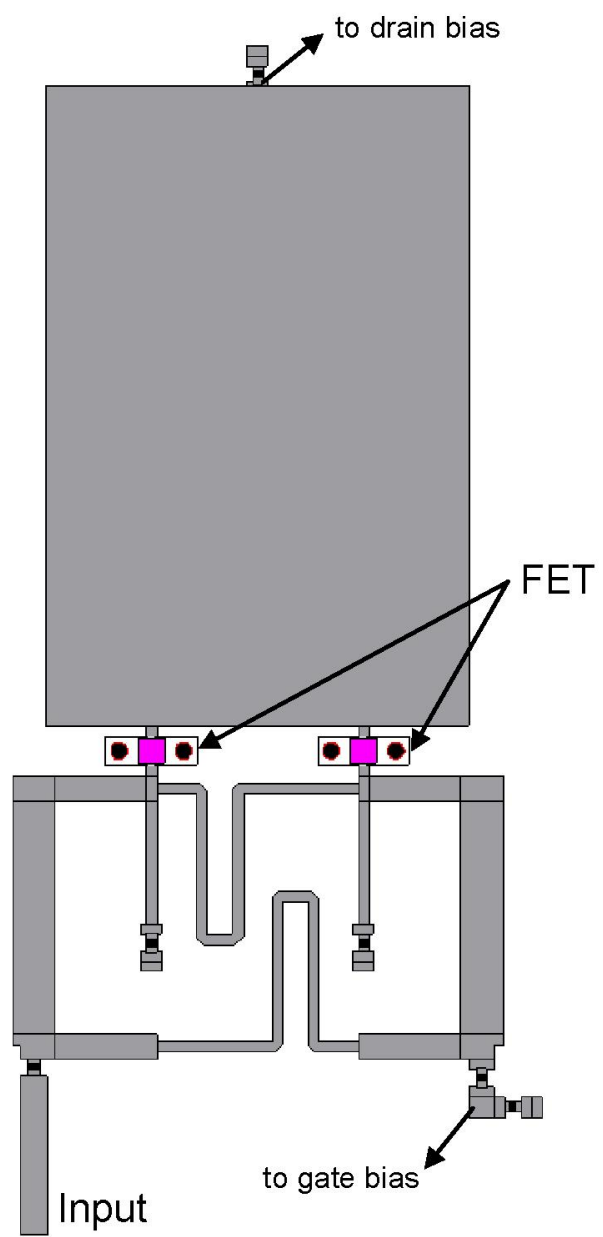

Fig. 6 Layout of $2.45 \mathrm{GHz}$ active antenna 
Both small-signal and harmonic balance (large-signal) simulations of the active antenna of Fig. 6 were performed using commercially available microwave circuit simulation software. The antenna was represented by its 3-port Sparameters up to $15 \mathrm{GHz}$ obtained from MoM EM simulations, and the FETs were represented by a non-linear equivalent circuit. The FETs have an $\mathrm{I}_{\mathrm{DSs}}$ of $60 \mathrm{~mA}$ and a $1 \mathrm{~V}$ knee voltage and were biased into class-A operation with: $V_{\mathrm{GS}}=-1$ and $V_{D S}=5 \mathrm{~V}$. Ideally, under this condition the output power of each FET is $18 \mathrm{dBm}$ and hence ideally the output power of the antenna should be $21 \mathrm{dBm}$. The DC conversion efficiency is expected to be $40 \%$.

Fig. 7 shows the small-signal active antenna input reflection coefficient frequency response and demonstrates that the active antenna is matched over a bandwidth sufficient for the $2.45 \mathrm{GHz}$ ISM band. It should be emphasized, that this match was obtained without matching the FET inputs and is therefore insensitive to component tolerances.

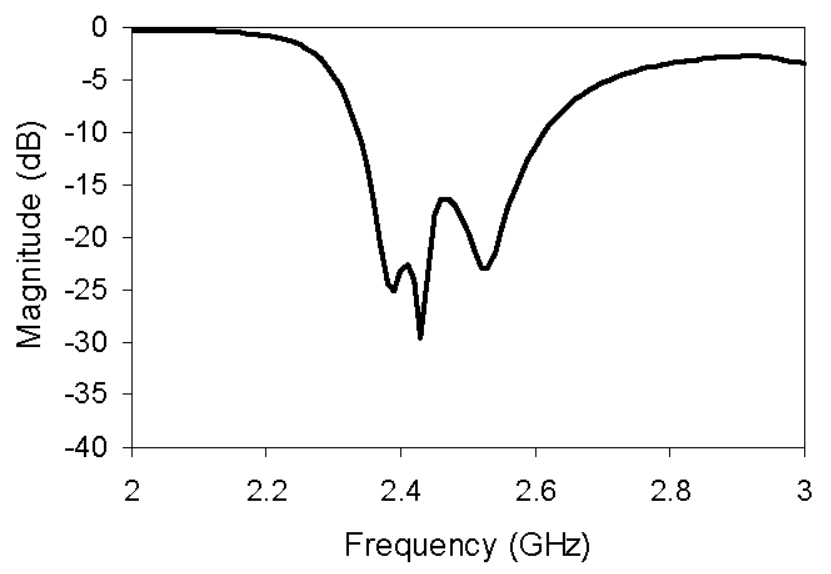

Fig. 7 Simulated active antenna small-signal input reflection coefficient.

Large-signal simulations were performed at frequencies covering the $2.45 \mathrm{GHz}$ ISM band and the best performance was obtained at $2.445 \mathrm{GHz}$ where the load-lines of each FET was identical and close to optimum for the bias operating point. The FET output capacitance is the main reason the active antenna centre frequency is slightly lower than that of the antenna on its own (Fig. 3). Fig. 8 shows the output power of both FETs and the antenna, and power-added-efficiency (PAE) of the amplifier at $2.445 \mathrm{GHz}$ as a function of the input power. It is clear from Fig 8, both FETs contribute equal output power and the total output power is twice the individual FET output power. The operating power gain is $12.8 \mathrm{~dB}$. The output power and efficiency is close to that ideally expected.

\section{CONCLULSION}

In this paper an active transmit antenna that combines two FET has been proposed. The novelty stems from the use of a modified branch-line coupler that achieves input matching without requiring FET input matching thereby reducing circuit sensitivity to FET parameter variations. The modified coupler drives the FETs equally but with a 180 degree phase difference and this enables linear polarization to be achieved when the FETs drains are directly connected to the non-radiating edge of a rectangular microstripline patch. The ability to place the FETs and input feed networks close to the antenna minimizes circuit size as will as minimize transmission lines losses. Simulations of $2.45 \mathrm{GHz}$ active transmit antenna demonstrate the feasibility of the proposed circuit.

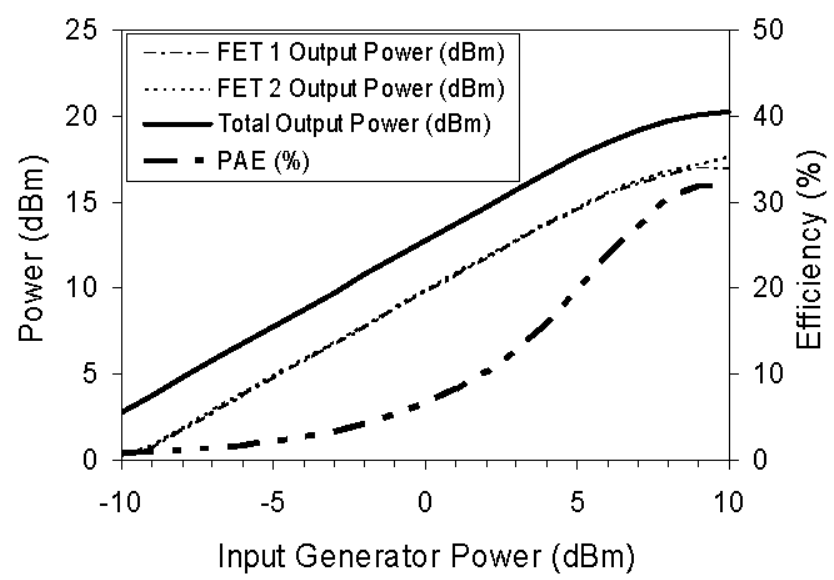

Fig. 8 Simulated large-signal performance of the active antenna at $2.445 \mathrm{GHz}$.

\section{REFERENCES}

[1] V. Radisic, Y. Qian and T. Itoh, "Novel Architectures for HighFrequency Amplifiers for Wireless Applications", IEEE Trans. on Microwave Theory and Techniques, 46(11), pp 1901 - 1909, Nov. 1998.

[2] C. Y. Hang, W. R. Deal, Y. Qian and T. Itoh, "High-Efficieincy PushPull Amplifier Integrated with Quasi-Yagi Antenna", IEEE Trans. on Microwave Theory and Techniques, 49(6), pp 1155 - 1161, June 2001.

[3] W. R. Deal, V. Radisic, Y. Qian and T. Itoh, "Integrated-Antenna PushPull Power Amplifiers", IEEE Trans. on Microwave Theory and Techniques, 47(8), pp 1418 - 1425, Aug 1999.

[4] S. Gao and P. Gardiner, IEEE Trans. on Microwave Theory and Techniques, 53(3), pp 1083 - 1088, March 2005.

[5] W. Wang and Y.P. Zhang, "0.18- $\mu \mathrm{m}$ CMOS Push-Pull Power Amplifier With Antenna in IC Package", IEEE Microwave and Wireless Component Letters, 14(1), pp 13 - 15, Jan 2004.

[6] C. A Balanis, Antenna Theory, Analysis and Design, $2^{\text {nd }}$ Ed., John Wiley and Sons, 1997, pp $722-779$.

[7] K. W. Eccleston, "Four-Way Power Combining Integrated Antenna", 2006 Asia Pacific Microwave Conference (APMC2006), 12 - 15 December 2006, Yokohama, Japan, pp $828-831$.

[8] C. C. Yang and W. S. Chan, "Linearly Polarized Intetegrated Antenna Using Quadrature Feed, Microwave and Optical Technology Letters, $27(5)$, pp $316-318,5$ Dec 2000.

[9] D. M. Pozar, Microwave Engineeering, $3^{\text {rd }}$ Edition, John Wiley and Sons, 2005.

[10] K. W. Eccleston, "Output Power Performance of Dual-Fed and SingleFed Distributed Amplifiers", Microwave and Optical Technology Letters, 27(4), pp 281 - 284, 20 November 2000. 